\title{
Pleural Effusion and Ascites Associated with Endometriosis-Mimicking Tuberculosis
}

\author{
Sara Marzook \\ Department of Obstetrics and Gynecology, King Abdul Aziz University Hospital, Jeddah, Saudi Arabia \\ Email: marzook330sara@gmail.com
}

How to cite this paper: Marzook, S. (2021) Pleural Effusion and Ascites Associated with Endometriosis-Mimicking Tuberculosis. Open Journal of Obstetrics and Gynecology, 11, 183-188. https://doi.org/10.4236/ojog.2021.112019

Received: January 27, 2021

Accepted: February 22, 2021

Published: February 25, 2021

Copyright $\odot 2021$ by author(s) and Scientific Research Publishing Inc. This work is licensed under the Creative Commons Attribution International License (CC BY 4.0).

http://creativecommons.org/licenses/by/4.0/

(c) (i) Open Access

\begin{abstract}
Objective: To report a case of Endometriosis associated with Pleural effusion. Design: Case report. Setting: Tertiary care center. Patient(s): A 30-year old woman presented with a right pleural effusion complicated with pneumothorax-mimicking TB. Intervention(s): Thoracentesis, pleural biopsy by a video-assisted thoracic surgery, pleurodesis, thoracic wedge resection, CT chest, CT Abdomen, and diagnostic Laparoscopy. Main Outcome Measure(s): After taking a GnRH analog, there was no recurrence of pleural effusion nor ascites. Result(s): Thoracentesis and wedge resection of lung ruled out malignancy. An omental mass biopsy obtained from diagnostic laparoscopy after the patient returned with drug-induced hepatitis, and ascites revealed endometriosis. Conclusion(s): Thoracic endometriosis is rare; however, it should be considered in the differential diagnosis by unknown causes of pleural effusion in reproductive age timeframe in women.
\end{abstract}

\section{Keywords}

Endometriosis, Pleural Effusion, Ascites, Pneumothorax

\section{Introduction}

Thoracic endometriosis is defined as the presence of endometrial glands and stroma abnormally implanted in the pleura and/or lungs. This form of endometriosis is rare and often diagnosed and managed rather late [1]. However thoracic cavity is the most common site of extra-pelvic endometriosis [2]. The pathogenesis of Endometriosis is not well understood. Thoracic endometriosis could be due to defect in the diaphragm that leads to entry of the endometrial tissue [3]. There is another theory known as coelomic Metaplasia where endometrial implants were identified in patients with absent Uterus in Mayer-RokitanskyKüster-Hauser syndrome [4]. The different forms of Thoracic endometriosis in- 
clude Catamenial pneumothorax, hemothorax, hemoptysis, lung nodules or non-catamenial [5].

Endometriosis is a benign estrogen-dependent condition with the presence of endometrial stroma and glands outside the uterine cavity. The endometrial implants are most commonly seen in the pelvis. The common clinical features and complications from endometriosis include dysmenorrhea, infertility, dyspareunia and chronic pelvic pain. The definitive diagnosis of endometriosis requires biopsy and histological confirmation of presence of endometrial stoma and glands. One of the treatment options in women who wish to preserve fertility involves suppression of gonadotropin release with $\mathrm{GnRH}$ agonists.

This case report was chosen to raise awareness of thoracic endometriosis and to consider it as a potential cause of recurrent pneumothorax, pleural effusion of unknown origin in reproductive age women.

In this case report, the patient was managed empirically with anti-tuberculosis medication until she developed side effects from the medication which lead to further investigations diagnosing Endometriosis. She presented with recurrent right pleural effusion complicated with pneumothorax.

\section{Case Report}

A 30-year old Nulliparous female with a recurrent right pleural Effusion complicated with Pneumothorax is the focus of this case report.

The patient complained of shortness of breath and productive cough for six months. In her medical history, there was no chest pain, hemoptysis, night sweats, or significant weight loss in the gynecological history there was history of primary infertility. Normal regular menstruation was present. There were no dyspareunia, abdominal pain, or bowel or urinary disturbance. No previous surgeries were conducted on the patient.

On examination, the patient's vitals were in the normal range besides being afebrile, conscious, and oriented. Oxygen saturation was maintained at room temperature. Normal BMI auscultation revealed decreased breath sound, S1 + S2 +0 no murmur. The abdomen was soft and lax, no palpable mass nor organomegaly or lower limb edema were detected.

Laboratory workup, including tumor markers such as Ca-125, was in the normal range. CT chest analysis showed rapid progression of right pleural effusion with mediastinal shift to the left side. No pulmonary nodules seen.The medical physicians referred the patient to thoracic surgery and a chest tube was inserted. The pleural fluid was sent for cytology exam, revealing scattered blood cells, reactive mesothelial cells, and no suspicious cells. Cultures of pleural fluid and sputum tested negative for acid-fast bacilli.

When the patient started to improve clinically the chest tube was removed however there was a re-expansion of the pleural effusion. It was then decided by the thoracic surgeon to performa pleural biopsy by video-assisted thoracic surgery, pleurodesis, and right lung wedge resection. The histopathology result 
showed unremarkable pink parenchyma with foci of hemorrhages, mild emphysematous changes, thickened visceral pleura. No evidence of granuloma, no evidence of malignancy.

The patient tolerated the procedure rather well however developed spike of fever postoperative with night sweats. Repeat pleural fluid culture was negative acid fast bacilli. She was referred to the infections disease team as a case of suspected Tuberculosis and was discharged empirically on anti tuberculosis medication. Upon discharge the night sweats subsided and she was afebrile.

Nine months later, the patient was presented to the emergency room with recurrent right pleural effusion and pneumothorax with ascites, abdominal tenderness and Jaundice. The diagnosis of drug induced hepatitis was made and anti-TB medication was stopped. Liver function tests gradually improved. CT abdomen was ordered which showed omental caking. The patient was referred to gynecological care, and diagnostic laparoscopy with biopsy of the omental mass was performed.

Histopathology results showed endometrial glands and stroma, while it yielded negative results for atypia and malignancy. The diagnosis of endometriosis was established. The Patient was followed up for 10 months since the first visit to the emergency department till diagnosis of thoracic endometriosis (from April 2019 till February 2020).

The patient was discharged on a gonadotropin-releasing hormone analog. After seven months, the patient mentioned no recurrence of ascites or pleural effusion. She is currently being managed in an IVF center to treat infertility (Table $1)$.

\section{Discussion}

The first documented case of bronchopulmonary endometriosis was in 1912 by Hart in Germany where he observed the pathology and spread of endometriosis based on the theory of benign metastasis [6].

Table 1. Demographic characteristics and clinical features of patient.

Clinical features presented by the patient Primary infertility

No Dysmenorrhea, no dyspareunia, no chronic pelvic or abdominal pain

Progressive shortness of breath, productive cough

Absent breath sounds on the right Lung

No hemoptysis, no weight loss
The sociodemographic characteristics

Nulliparous

Saudi Arabian

30 years of age

Housewife

Clinical features after developing drug induced hepatitis include: abdominal tenderness, ascites, nausea and vomiting. Jaundice and dark urine present.

Family history of breast cancer (mother) 
The most common presentation of thoracic endometriosis is catamenial pneumothorax which is defined as at least two episodes of recurrent spontaneous pneumothorax occurring one day before menses or 72 hours before the onset of menses [1] [7] [8] [9]. However, there is also endometriosis-related noncatamenial pneumothorax [10] [11]. In the study by Marco Alifano [5], they mentioned that $12.8 \%$ of cases were endometriosis-related non catamenial pneumothorax. Besides, there was a higher prevalence of the presence of pneumothorax on the right side [5] which is also seen in this patient. In the series report done by Legras, there was $41 \%$ endo metriosis-related and/or catamenial pneumothoraces [12]. In this case report, there was no relation of the patient's recurrent pleural effusion and pneumothorax to her onset of menses. In a case report by Parisa [1] the patient was 48 years old presented with thoracic endometriosis and bowel obstruction due to the endometriosis. The 48 year old was then managed with hysterectomy and bilateral salpingectomy. The patient presented in this case report was much younger and wanted to preserve her fertility. No signs of bowel obstruction were seen.

There was progressive shortness of breath and cough with absence of hemoptysis in this patient. In the case report by Schwartz the patient during menses had hemoptysis [13].

The patient in this case had clinical and radiological ascites after readmittance because of drug-induced hepatitis. The presence of ascites arises suspicion of malignancy though it can be also associated with endometriosis [14] [15] [16]. In the case report by I.O. Olubuyide mentioned a 19 year old with massive ascites, dysmenorrhea, weight loss and right atelectasis on chest $\mathrm{x}$ ray. The patient in his case report had also negative culture for acid fast bacilli. Laparotomy then revealed enlarged ovaries. Lesions were biopsied and histopathology revealed endometriosis [16].

When mesothelial cells are irritated, a raise in ca-125 occurs that is not specific to endometriosis [8]. The Lab investigations can show elevated Ca-125 in endometriosis which also raises suspension of ovarian malignancy [17] [18] [19]; however, in this case report, the patient's tumor markers Ca-125 and Ca 19-9 were all in the normal range. In Bagan reference [19] there was 29 percent raise in the ca-125 marker in patients with thoracic endometriosis.

The diagnosis of thoracic endometriosis in histopathology is difficult. This is known as probable thoracic endometriosis where the tissue within the thorax is suggestive, but not definitively diagnostic, of endometriosis [12]. The histopathology after wedge resection and pleural biopsy in this patient did not give a histopathology result suggestive of endometriosis.

Most symptoms of endometriosis involve menstruation in which the pelvic organs, such as ovaries and fallopian tubes, are afflicted, causing menstrual disturbances such as dysmenorrhea.

Other findings associated with endometriosis are infertility and endometrioma [16] [19]. In a study of 1000 women with endometriosis, it was found that 
$80 \%$ presented with dysmenorrhea, $25 \%$ with infertility, and $20 \%$ with endometrioma. It is still unknown how the endometrial tissue reaches the thorax [5]. Endometriosis affects $5 \%-15 \%$ of women in the reproductive age group and many are asymptomatic. In the previously reported cases, the conservative management of endometriosis would be giving $\mathrm{GnRH}$ analogs [20].

This case report points out the complexity of diagnosing endometriosis. This condition should be a part of the differential diagnosis when a patient in the reproductive age group has recurrent spontaneous pneumothorax and pleural effusion. Increased awareness in reproductive age women with repeated negative acid fast bacilli culture and malignancy ruled out to look for Endometriosis being the possible cause of her clinical features.

\section{Conflicts of Interest}

The author declares no conflicts of interest regarding the publication of this paper.

\section{References}

[1] Parisa, A. and David, C. (2014) Thoracic Endometriosis Syndrome. The Permanente Journal, 18, 61-65.

[2] Bagan, P., Berna, P., Assoud, J., Hupertan, V., Le Pimpec Barthes, F. and Riquet, M. (2008) Value of Cancer Antigen Ca-125 for Diagnosis of Pleural Endometriosis in Females with Recurrent Pneumothorax. European Respiratory Journal, 31, 140-142. https://doi.org/10.1183/09031936.00094206

[3] Alifano, M., Trisolini, R., Cancellieri, A. and Regnard, J.F. (2006) Thoracic Endometriosis: Current Knowledge. The Annals of Thoracic Surgery, 81, 761-769.

https://doi.org/10.1016/j.athoracsur.2005.07.044

[4] Mok-Lin, E.Y., Wolfberg, A., Hollinquist, H. and Laufer, M.R. (2010) Endometriosis in a Patient with Mayer-Rokitansky-Küster-Hauser Syndrome and Complete Uterine Agenesis: Evidence to Support the Theory of Coelomic Metaplasia. Journal of Pediatric and Adolescent Gynecology, 23, e35-e37. https://doi.org/10.1016/j.jpag.2009.02.010

[5] Alifano, M., Jablonski, C., Kadiri, H., Falcoz, P., Gompel, A., Camilleri-Broet, S., et al. (2007) Catamenial and Noncatamenial, Endometriosis-Related or Nonendometriosis-Related Pneumothorax Referred for Surgery. American Journal of Respiratory and Critical Care Medicine, 176, 1048-1053. https://doi.org/10.1164/rccm.200704-587OC

[6] Hart, C. (1912) Histologically Benign Metastasis from the Formation of Adenomyoma 22 Years after the Extirpation of a Tumor of the Genitals. Frankfurter Zeitschrift für Pathologie, 10, 78-90.

[7] Peikert, T., Gillespie, D.J. and Cassivi, S.D. (2005) Catamenial Pneumothorax. Mayo Clinic Proceedings, 80, 677-680. https://doi.org/10.4065/80.5.677

[8] Jubanyik, K.J. and Comite, F. (1997) Extrapelvic Endometriosis. Obstetrics and Gynecology Clinics of North America, 24, 411-440. https://doi.org/10.1016/S0889-8545(05)70311-9

[9] Fukuda, S., Hirata, T., Nweiishi, K., et al. (2018) Thoracic Endometriosis Syndrome: Comparison between Catamenial Pneumothorax or Endometriosis-Related Pneu- 
mothorax and Catamenial Hemoptysis. European Journal of Obstetrics \& Gynecology and Reproductive Biology, 225, 118-123. https://doi.org/10.1016/j.ejogrb.2018.04.021

[10] Simpson, A. and Skelly, E. (2008) Catamenial Pneumothorax. Emergency Medicine Journal, 25, 859. https://doi.org/10.1136/emj.2008.062679

[11] Legras, A., Mansuet-Lupo, A., Rousset-Jablonski, C., et al. (2014) Pneumothorax in Women of Child-Bearing Age: An Update Classification Based on Clinical and Pathologic Findings. Chest, 145, 354-360. https://doi.org/10.1378/chest.13-1284

[12] Guo, S.W. (2009) Recurrence of Endometriosis and Its Control. Human Reproduction Update, 15, 441-461. https://doi.org/10.1093/humupd/dmp007

[13] Counsellor, V.S. (1938) Endometriosis. A Clinical and Surgical Review. American Journal of Obstetrics and Gynecology, 36, 887.

[14] Iwasaka, T., Okuma, Y., Yoshimura, T., Kidera, Y. and Sugimori, H. (1985) Endometriosis Association with Ascites. Obstetrics \& Gynecology, 66, 72S-75S.

[15] Meyers, T.J., Arena, B. and Grania, C.O. (1995) Pelvic Endometriosis Mimicking Advanced Ovarian Cancer: Presentation with Pleural Effusion, Ascites, Elevated CA 125 Level. American Journal of Obstetrics and Gynecology, 173, 966-967. https://doi.org/10.1016/0002-9378(95)90381-X

[16] Olubuyide, I.O., Adebajo, A.O., Adeleye, J.A. and Solanke, T.F. (1988) Massive Ascites Associated with Endometrioma in Nigerian African. International Journal of Gynecology \& Obstetrics, 27, 439-441. https://doi.org/10.1016/0020-7292(88)90127-0

[17] Tsunezuka, Y., Sato, H., Kodama, T., Shimizu, H. and Kurumaya, H. (1999) Expression of Ca-125 in Thoracic Endometriosis in a Patient with Catamenial Pneumothorax. Respiration, 66, 470-472. https://doi.org/10.1159/000029414

[18] El-Newihi, H.M., Antaki, J.P., Rajan, S. and Reynolds, T.B. (1995) Large Bloody Ascites in Association with Pelvic Endometriosis. The American Journal of Gastroenterology, 90, 632-634.

[19] Sinaii, N., Plumb, K., Cotton, L., Lambert, A., Kennedy, S., Zondervan, K., et al. (2008) To Determine the Relationship between Disease Severity and Patient Characteristics in Endometriosis. Fertility and Sterility, 89, 538. https://doi.org/10.1016/j.fertnstert.2007.03.069

[20] Conn, P.M. and Crowley, W.F. (1991) Gonadotropin-Releasing Hormone and Its Analogues. The New England Journal of Medicine, 324, 93-103. https://doi.org/10.1056/NEJM199101103240205 